\title{
Erratum: Influence of minivalleys and Berry curvature on electrostatically induced quantum wires in gapped bilayer graphene [Phys. Rev. B 98, 155435 (2018)]
}

Angelika Knothe and Vladimir Fal'ko

(Received 20 June 2019; published 16 July 2019)

DOI: 10.1103/PhysRevB.100.049903

We would like to correct the following typographical errors in the paper:

Page 1, Eq. (1):

There is a digit missing in the value of the $\gamma_{1}$ parameter. It should read $\gamma_{1}=0.38 \mathrm{meV}$.

Page 2, Eq. (4):

The minus sign in the definition of the orbital magnetic moment is a reminiscence of a missing fraction $\frac{1}{2}$ that has been mistyped in latex. The correct equation should read

$$
M=-\frac{1}{2} i e \hbar\left\langle\nabla_{\mathbf{p}} \Phi(\mathbf{p})|\times[\epsilon(\mathbf{p})-H(\mathbf{p})]| \nabla_{\mathbf{p}} \Phi(\mathbf{p})\right\rangle \cdot \mathbf{e}_{z} .
$$

Also, the indication of the sign of the elementary charge has been used inconsistently. So it should read $e<0$ under Eq. (4). This effects the analytical formulas for $M$ in the following, which should read with the opposite sign: $M \approx-\epsilon(\mathbf{p}) \frac{e}{\hbar} \Omega=\xi \frac{e \hbar}{2 m} \frac{p^{2}}{2 m} \frac{\Delta}{\epsilon(\mathbf{p})^{2}}$.

Page 2, Fig. 2: The scale bar in the top left panel of Fig. 2 should be indexed with $0.1 \hbar \mathrm{nm}^{-1}$ (instead of $1 \hbar \mathrm{nm}^{-1}$ ).

Page 6, Eq. (10):

Likewise, the signs of $\Omega$ and $M$ must be adjusted correspondingly

$$
\begin{aligned}
\Omega & =-\xi \frac{\hbar^{2}}{2 m} \frac{p^{2}}{2 m} \frac{\Delta}{\epsilon(\mathbf{p})^{2}}, \\
M & \approx-\epsilon(\mathbf{p}) \frac{e}{\hbar} \Omega \\
& =\xi \frac{e \hbar}{2 m} \frac{p^{2}}{2 m} \frac{\Delta}{\epsilon(\mathbf{p})^{2}} .
\end{aligned}
$$

None of the results or the statements of the paper are affected by these corrections. 